\title{
Underground experiments and their impact on stellar modeling
}

\author{
Gianluca Imbriani*'and Antonino Di Leva \\ Physics Department, University of Naples Federico II and INFN section of Naples, 80126 \\ Naples, Italy \\ E-mail: gianluca.imbriani@unina.it
}

Low-energy studies of thermonuclear reactions in a laboratory at the earth's surface are hampered predominantly by background induced by cosmic rays in the detectors, leading typically to more than 10 events per hour in common detectors. Conventional passive or active shielding around the detectors can only partially reduce the problem. The best solution so far was to install an accelerator facility in a deep underground laboratory, in a similar way to solar neutrino detectors. In the case of National Laboratory of Gran Sasso the $1400 \mathrm{~m}$ of rock above the laboratory halls leads to a reduction of the muon flux, the most penetrating component of the cosmic-rays, by a factor $10^{6}$ with respect to the Earth's surface.

Several cross sections belonging to hydrogen burning have been studied in the framework of this unique project, called LUNA (Laboratory for Underground Nuclear Astrophysics), that was initiated almost 20 years ago.

The most important results achieved and their impact on stellar evolution and nucleosynthesis modeling will be reviewed.

XII International Symposium on Nuclei in the Cosmos,

August 5-12, 2012

Cairns, Australia

\footnotetext{
${ }^{*}$ Speaker.

${ }^{\dagger}$ The work described in this paper has been mainly done in the framework of the LUNA experiment. The authors would thank all the members of this international collaborations (http://luna.lngs.infn.it/collaboration.php).
} 


\section{Introduction}

The detailed description of most astrophysical and cosmological scenarios requires an accurate knowledge of the many relevant nuclear processes involved. Nuclear fusion reactions are at the heart of nuclear astrophysics as they determine the nucleosynthesis of the elements in the earliest stages of the universe and in all the objects formed thereafter, and control the energy generation, neutrino luminosity, and evolution of stars. An accurate and precise knowledge of the cross section of these reactions is thus essential to describe the evolution of the universe. Since most of the cross sections at the astrophysical relevant energies are too small to be directly measured in a laboratory, they need to be extrapolated through models on the basis of the existing experimental data at higher energies, e.g. using $R$-matrix fits [1]. In many cases, because of the difficulty of taking into account all of the complexity of the nuclear structure, the accuracy reached by extrapolation is not sufficient to obtain unambiguous results from models. Improved measurements on a wide range of energies are needed to reduce the uncertainty of the extrapolations.

In the stellar environment the energy available to nuclei is much lower than the Coulomb barrier, i.e. the nuclear reactions take place via quantum tunneling effect. For example, the core temperature of the Sun is about $15 \cdot 10^{6} \mathrm{~K}$ and, therefore, the mean kinetic energy available to protons is about $1 \mathrm{keV}$, much lower than the $550 \mathrm{keV}$ of the Coulomb barrier.

For charged particle reactions it is customary to write the cross section as

$$
\sigma(E)=\frac{S(E)}{E} e^{-2 \pi \eta}
$$

where the exponential term takes into account the s-wave tunneling probability, $\eta$ is the Sommerfeld parameter and the $S$-factor, $S(E)$, includes all the nuclear properties of the reaction and is a smoothly varying function in energy for non resonant reactions $[2,3]$. In a stellar plasma, to obtain the reaction rate, the number of reactions per second, one has to calculate the averaged product of the cross section and the relative velocity distributed according to the Maxwell-Boltzmann distribution

$$
\langle\sigma v\rangle=\sqrt{\frac{8}{\pi \mu}} \frac{1}{(k T)^{3 / 2}} \int_{0}^{\infty} \frac{S(E)}{E} e^{-2 \pi \eta} e^{-E / k T} d E,
$$

where $k$ is the Boltzmann constant. While both the energy distribution function and the tunneling probability through the Coulomb barrier are small, the convolution of the two functions results in a peak (the Gamow peak) near the energy $E_{0}=\left(\frac{b k T}{2}\right)^{2 / 3}$, with $b=0.989 Z_{1} Z_{2} \mu^{1 / 2}$, giving a sufficiently high probability to allow a significant number of reactions to occur.

For a given stellar temperature $T$ the energy corresponding to the Gamow peak, that is larger than $k T$, are typically in the order of tens of $\mathrm{keV}$. Most of the reactions between light nuclei at such energies have cross sections of the order of $10^{-9}-10^{-12}$ barn. These correspond, under typical laboratory conditions, to experimental counting rates ranging from a few events per day to a few events per month. This means that in realistic experimental conditions, the expected counting rate is prohibitively low and the competition with the background induced in the detectors by the cosmic rays strongly hinders the possibility of obtaining statistically significant results.

Besides the cosmic rays, other sources of background must be considered: the environmental radioactivity, i.e. radionuclides belonging to the natural radioactive series of uranium and thorium 
or long-lived natural radionuclides such as ${ }^{40} \mathrm{~K}$, and the beam induced background, i.e. nuclear reactions induced by the beam on target impurities or other parts of the experimental setup. Each of these sources produces background with characteristic energy ranges and rates, so that each reaction studied needs special attention in suppressing the relevant background component. A number of techniques have been developed to reduce the background component by improved detector configuration, event identification and background rejection techniques $[4,5]$.

A major step forward was the Laboratory for Underground Nuclear Astrophysics (LUNA), a low energy underground accelerator facility at the Gran Sasso laboratory of INFN [6]. LUNA is at present still a worldwide unique facility for the measurement of low energy cross sections of astrophysical interest.

\section{Why going underground}

Low-energy studies of thermonuclear reactions in a laboratory on the earth's surface are hampered predominantly by background effects of cosmic rays in the detectors, leading typically to more than 10 events per hour in common detectors. Conventional passive or active shielding around the detectors can only partially reduce the problem. The best solution is to install an accelerator facility in a laboratory deep underground, in a similar way to the solar neutrino detectors.

The experimental halls of Laboratori Nazionali del Gran Sasso (LNGS) in Italy, completed in 1987, are placed below an average thickness of $1400 \mathrm{~m}$ of rock (3800 metre water equivalent). This leads to a reduction of the muon flux, the most penetrating component of the cosmic-rays, by a factor $10^{6}$ compared to the surface, thus providing a low background environment [6]. For a Ge $\gamma$-ray detector this corresponds to a reduction in the $\gamma$-ray background of about 3 orders of magnitude in the energy region above the natural radioactivity, i.e. $E_{\gamma}>2.6 \mathrm{MeV}$, see figure 1 .

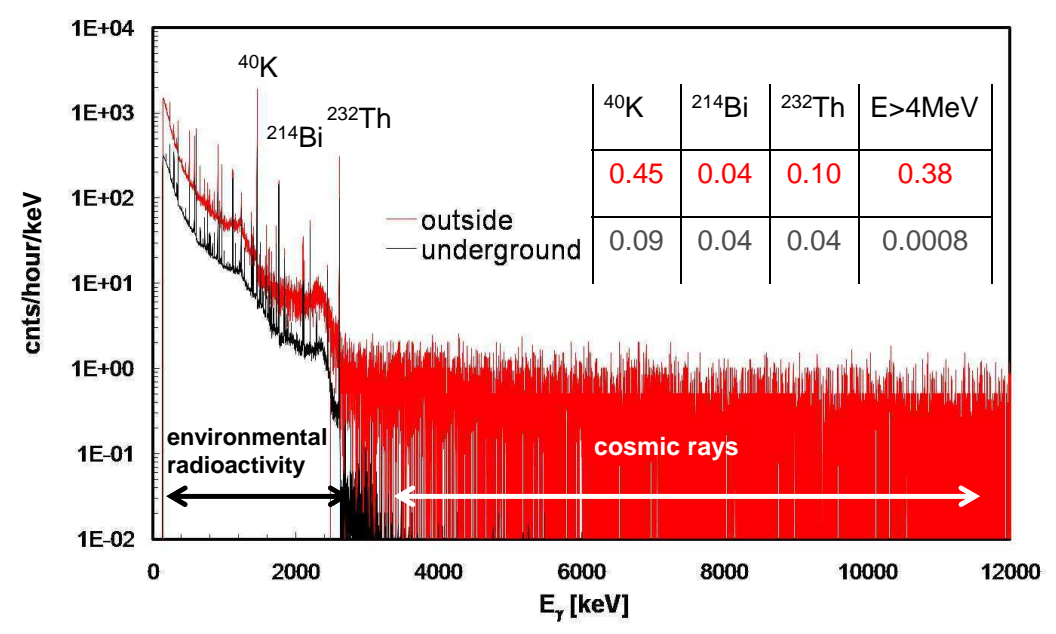

Figure 1: $\gamma$-ray background as observed with a HPGe detector in a surface laboratory (red), compared with one acquired underground (black).

In a surface laboratory a passive shielding, such as lead, is usually placed around the detectors to suppress the background, nevertheless its efficiency is limited to a certain thickness. In 


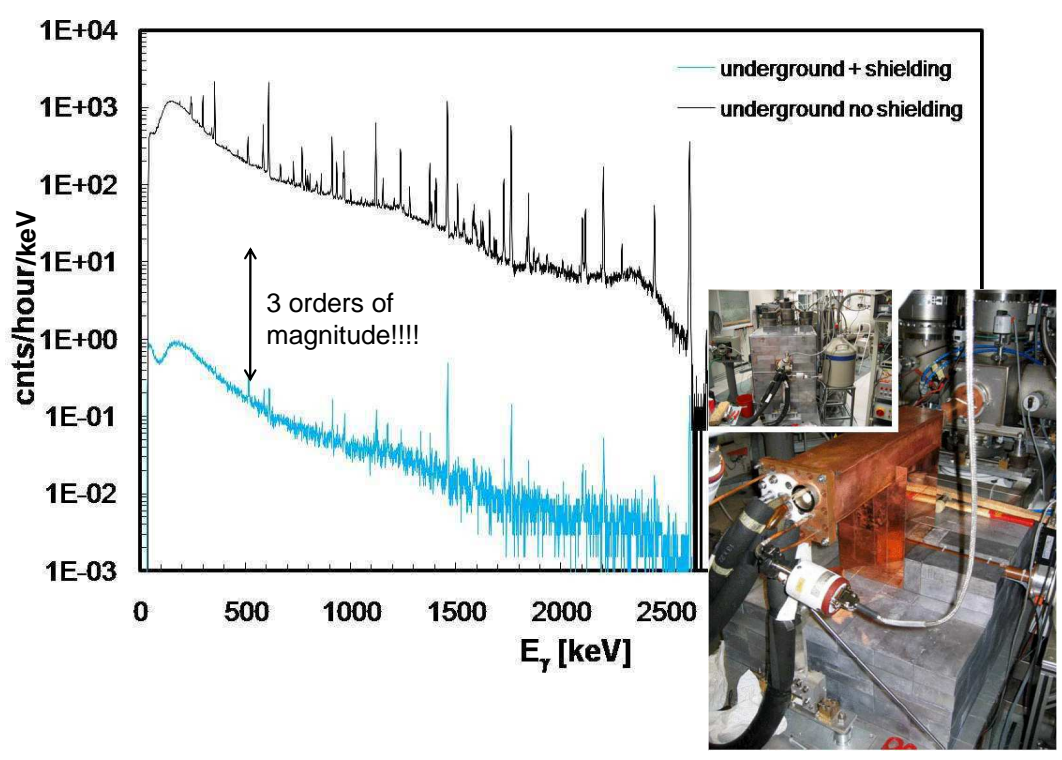

Figure 2: Underground $\gamma$-ray backgrounds as observed with a HPGe detector non shielded (black line) and with a lead shielding and radon box (blue line) [7].

fact cosmic muons interact with the shielding material creating energetic neutrons which, in turn, induce nuclear reactions becoming a new secondary source of background $\gamma$-rays. Clearly, this background component is dramatically reduced if the muon flux is significantly suppressed, as in an underground laboratory, and then the shielding is very effective in reducing also the natural radioactivity background. A special care is needed for radon ${ }^{222} \mathrm{Rn}$, which is a noble gas and a short-lived radioactive product of the uranium and thorium decay chains that diffuses through the shielding. A popular solution to this problem is to house the detector in a box with a little overpressure of flushing dry gas $[8,9]$, see figure 2 .

The interaction of the ion beam with traces of other elements present in the target and/or parts of the experimental setup, may give rise to beam induced background, which is specific for each experiment. Under this respect each reaction must be considered individually taking into account its $Q$-value, the resonance structure of the possible background reactions, the beam energy and the particle spectrum involved. The choice of the type of target (gaseous or solid) and the target production process are of fundamental importance.

High resolution spectra from Ge detectors make background recognition and identification easier and more reliable and provide an excellent signal to background ratio. However, most detectors with good resolution provide only a limited efficiency with respect to high density materials like $\mathrm{BGO}$ or $\mathrm{LaBr}$, that might be needed to achieve sufficient statistics.

\section{H-burning in stars}

Most of the results achieved by the LUNA experiment in the last 20 years led to a better understanding of the H-buring phase in stars. As a pilot project for an underground accelerator facility, a 
home-made $50 \mathrm{kV}$ accelerator was installed at LNGS in $1990[10,6]$. During this first phase of the experimental activities, LUNA I, the cross section of the nuclear processes ${ }^{3} \mathrm{He}\left({ }^{3} \mathrm{He}, 2 \mathrm{p}\right){ }^{4} \mathrm{He}[11]$, $\mathrm{d}\left({ }^{3} \mathrm{He}, \mathrm{p}\right)^{4} \mathrm{He}[12,13]$ and $\mathrm{d}(\mathrm{p}, \gamma)^{3} \mathrm{He}[14]$ were measured. For the the first time ever the astrophysical relevant energy of the solar Gamow peak was experimentally reached.

Supported by these scientific successes the collaboration could move to a second phase LUNA II, installing a new accelerator, a commercial high-current $400 \mathrm{kV}$ machine. It can provide on target proton beam currents up to $500 \mu \mathrm{A}$ and He-beam current up to $1 \mathrm{~mA}[15,6]$. This opened the possibility of studying other key reactions of the hydrogen burning phases like ${ }^{3} \mathrm{He}\left({ }^{4} \mathrm{He}, \gamma\right)^{7} \mathrm{Be}$, ${ }^{14} \mathrm{~N}(\mathrm{p}, \gamma){ }^{15} \mathrm{O},{ }^{15} \mathrm{~N}(\mathrm{p}, \gamma){ }^{16} \mathrm{O}$ and ${ }^{25} \mathrm{Mg}(\mathrm{p}, \gamma){ }^{26} \mathrm{Al}$.

The hydrogen burning phase is initiated when the center of a star reaches a temperature of about $6 \mathrm{MK}$ and the proton-proton reaction can efficiently synthesize deuterium. In the hydrogen rich environment the deuterium reacts almost instantly with another proton producing ${ }^{3} \mathrm{He}$, which in turn forms, together with another ${ }^{3} \mathrm{He}$ nucleus, an $\alpha$ particle and releases two protons through ${ }^{3} \mathrm{He}\left({ }^{3} \mathrm{He}, 2 \mathrm{p}\right){ }^{4} \mathrm{He}$. This represents the main termination of the pp-chain with a net conversion of $4 \mathrm{p}$ $\rightarrow{ }^{4} \mathrm{He}+2 e^{+}+2 v_{e}+26.73 \mathrm{MeV}$. The other branch of the pp-chain proceeds through the reaction ${ }^{3} \mathrm{He}\left({ }^{4} \mathrm{He}, \gamma\right){ }^{7} \mathrm{Be}$ with an additional branching through the electron capture of ${ }^{7} \mathrm{Be}$ or a subsequent proton capture followed by the $\beta$ delayed $\alpha$ decay of ${ }^{8} \mathrm{~B}$, resulting in two ${ }^{4} \mathrm{He}$ nuclei. The final result is always the transformation of four protons into a helium nucleus accompanied by the emission of neutrinos.

In more massive stars, $M_{\mathrm{star}}>1.5 M_{\odot}$, the core reaches temperature higher than $15 \mathrm{MK}$. In this case, the carbon nuclei present in the core of the star can react efficiently with the protons, producing ${ }^{13} \mathrm{~N}$. This reaction starts the so called CNO-cycle. After a series of proton captures and decays [16] four protons are transformed again into a helium nucleus with the emission of two neutrinos. At this temperature the rate of energy generation by the CNO-cycle is larger than that by the ppchain; moreover, its temperature dependence is much steeper. The number of carbon and nitrogen nuclei remains constant through the cycle. The ${ }^{14} \mathrm{~N}(\mathrm{p}, \gamma){ }^{15} \mathrm{O}$ has the lowest cross section among the reactions in the cycle, therefore at the end of this H-burning phase, almost all carbon is transformed into nitrogen.

In the next sections we summarize the results of the measurements important in the framework of the solar neutrino flux determination and those important in $\mathrm{CNO}$ burning and the shell hydrogen burning.

\subsection{H-burning in the sun: solar neutrino}

The presence of a low energy resonance in the ${ }^{3} \mathrm{He}\left({ }^{3} \mathrm{He}, 2 \mathrm{p}\right)^{4} \mathrm{He}$ was considered as a possible nuclear explanation for the deficit of observed neutrinos from the sun, the solar neutrino problem. The LUNA experiment measured its cross section down to about $15 \mathrm{keV}$ [11], thus fully covering the solar Gamow peak. The results excluded the existence of any resonance in that energy region. This important result showed that the solution of the solar neutrino problem was not in the uncertainties of the Standard Solar Model, but must have been searched elsewhere. The successful experiments of the Sudbury Neutrino Observatory (SNO) and KamLAND experiments [17, 18] proved the neutrino oscillation and gave an explanation of the observed solar neutrino fluxes in Earth neutrino detectors. This in turn opened a new era of neutrino spectroscopy, in which the solar neutrino fluxes can be used to investigate the standard model of particle physics. 
The nuclear cross section of ${ }^{3} \mathrm{He}\left({ }^{4} \mathrm{He}, \gamma\right){ }^{7} \mathrm{Be}$ is, presently, one of the largest uncertainties in the prediction of the solar neutrino flux [19]. The ${ }^{3} \mathrm{He}\left({ }^{4} \mathrm{He}, \gamma\right){ }^{7} \mathrm{Be}$ reaction has also important implications on Big Bang nucleosynthesis (BBN), where the predicted abundance of ${ }^{7} \mathrm{Li}$ is a factor of 2 to 3 larger than observation. A precision determination of this cross section is the basis to investigate possible solutions of the ${ }^{7} \mathrm{Li}$ problem.

During the last decades, many efforts have been devoted to the determination of this cross section. The experiments exploited the detection of prompt $\gamma$-rays as well as the radioactive decay of the ${ }^{7} \mathrm{Be}\left(\mathrm{T}_{1 / 2}=53\right.$ days), while in a few cases, including the measurement at LUNA, both techniques were used [7, 20, 21, 22, 23]. The LUNA measurements were performed at $E_{\mathrm{cm}}$ between 93 and $170 \mathrm{keV}$. The prompt $\gamma$-rays of the ${ }^{3} \mathrm{He}\left({ }^{4} \mathrm{He}, \gamma\right){ }^{7} \mathrm{Be}$ reaction $(\mathrm{Q}=1.586 \mathrm{MeV})$ are located in the region of natural background. As mentioned in section 2, passive lead shielding is very effective in an underground laboratory, therefore a massive shield was built around both the target chamber and the detector. Figure 3 compares a background spectrum with the prompt $\gamma$-ray spectrum at the lowest measurement energy of $E=93 \mathrm{keV}$.

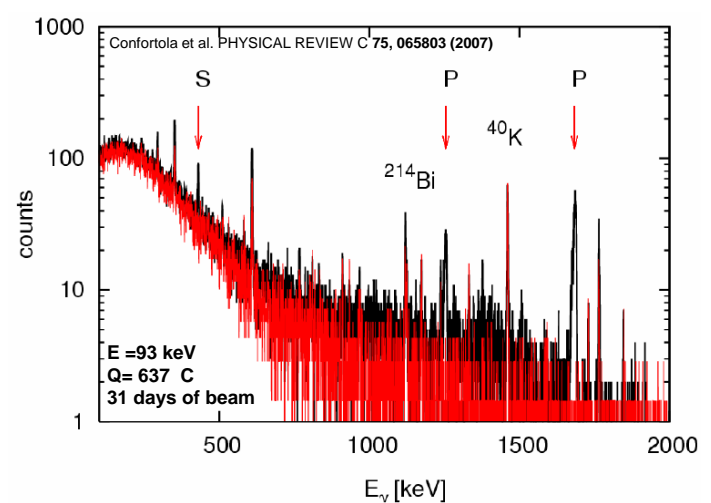

Figure 3: ${ }^{3} \mathrm{He}\left({ }^{4} \mathrm{He}, \gamma\right){ }^{7} \mathrm{Be}$ prompt $\gamma$-ray spectrum at $E_{\mathrm{cm}}=93 \mathrm{keV}$ (black) compared with natural radioactivity background (red line), from [7]. The arrows indicate primary $(\mathrm{P})$ and secondary $(\mathrm{S})$ transitions.

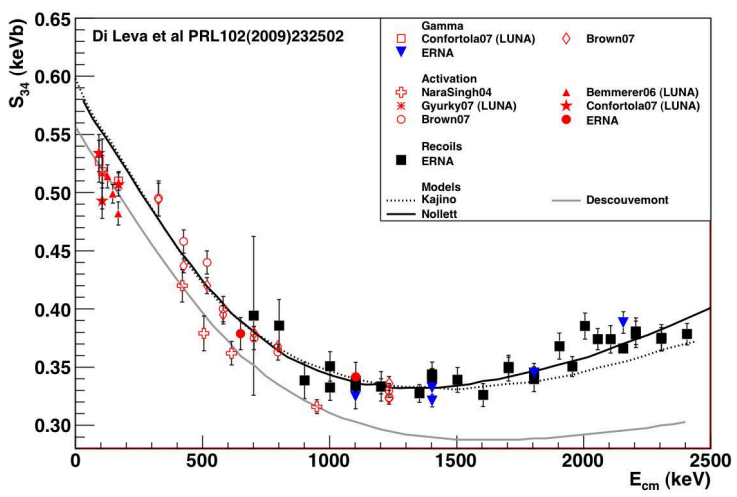

Figure 4: Comparison, from [24], of the results of the modern experimental data with different model calculations fitted at $E<2 \mathrm{MeV}$ (solid black line [25], solid grey line [26], dotted line [27]).

The results from the two methods do not show any discrepancy at the level of the achieved accuracy (4\%). Agreement between the two techniques was also found by a recent experiment [20] where the ${ }^{3} \mathrm{He}\left({ }^{4} \mathrm{He}, \gamma\right){ }^{7} \mathrm{Be}$ reaction was measured in the energy region between $E_{\mathrm{cm}}=0.35$ and $1.2 \mathrm{MeV}$. These experiments covered the energy range of $\mathrm{BBN}(180 \mathrm{keV}<E<40 \mathrm{keV})$, on the other hand the Gamow energy $\left(E_{0}=22 \mathrm{keV}\right)$ in the Sun was not reached, and models have to be used to extrapolate the data. Recently a new determination of this cross section has been obtained by the direct detection of the ${ }^{7} \mathrm{Be}$ recoils using the recoil separator ERNA [24]. The total cross section was measured in the energy range $E=0.7-3.2 \mathrm{MeV}$. In comparison with previous works, a significant discrepancy in both the absolute scale and the energy dependence of the $S$-factor was found in the energy range $E>1.0 \mathrm{MeV}$. On the basis of these data a selection among the several theoretical models available can be done, see figure 4, to obtain more robust extrapolations to solar Gamow energy. Nevertheless still the best estimates suffer an uncertainty of about $8 \%$, still larger than the precision level required by the astrophysics community [24, 19]. 


\subsection{CNO burning: Age of Globular Clusters and C production in AGB}

The measurement of the ${ }^{14} \mathrm{~N}(\mathrm{p}, \gamma){ }^{15} \mathrm{O}(Q=7.297 \mathrm{MeV})$ cross section, initiated the study of reactions involved in the $\mathrm{CNO}$ cycle. This reaction is the slowest reaction in the $\mathrm{CN}$ cycle and therefore is the key to understand the timescale, as well as the overall energy production and neutrino production, of the $\mathrm{CNO}$ burning [28].

The measurement of the ${ }^{14} \mathrm{~N}(\mathrm{p}, \gamma){ }^{15} \mathrm{O}$ performed at LUNA had two phases. The first part concentrated on the measurement of the yield of all $\gamma$-transitions using a HPGe-detector [29, 30, 31]. The primary $\gamma$-rays have energies higher than $5 \mathrm{MeV}$, so a clean signal could be detected only thanks to the background free energy spectra, from $E_{\mathrm{cm}}=360 \mathrm{keV}$ down to $110 \mathrm{keV}$, see figure 5 . To push the cross section measurements toward lower energies, a second phase of the experiment was performed using a nearly $4 \pi$ BGO summing crystal $[32,33]$. The absolute efficiency at $7 \mathrm{MeV}$ was about $65 \%$ that allowed to push the measurements of the total cross section down to $70 \mathrm{keV}$. This energy is at the upper edge of the solar Gamow peak, $E_{0}=27 \mathrm{keV}$, but is within the Gamow peak of AGB shell hydrogen burning. The two different approaches were complementary and both took extreme advantage of the low background laboratory, see figure 6 . It should be pointed out that the reliable extrapolation of the LUNA data to the solar Gamow peak required the combination of low-energy and data at higher energies, namely the results of the LENA experiment at TUNL [5]. These experiments cover an energy range from 70 to $480 \mathrm{keV}$. Additional information is provided by experiments which used indirect methods such as the Doppler shift attenuation method [34, 35], Coulomb excitation [36] and asymptotic normalization coefficients (ANC) [37]. Nevertheless the uncertainty on data at high energy affects the low energy extrapolation. This calls for improved measurements in the energy range above $500 \mathrm{keV}$ [1].

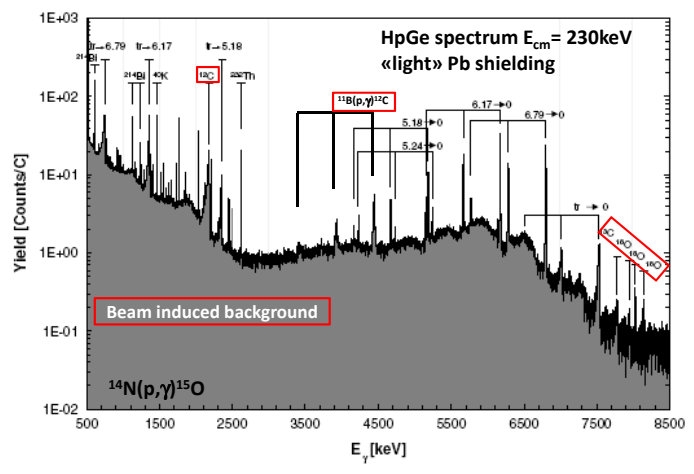

Figure 5: Spectrum taken during the ${ }^{14} \mathrm{~N}(\mathrm{p}, \gamma){ }^{15} \mathrm{O}$ experiment at $230 \mathrm{keV}$ [29].

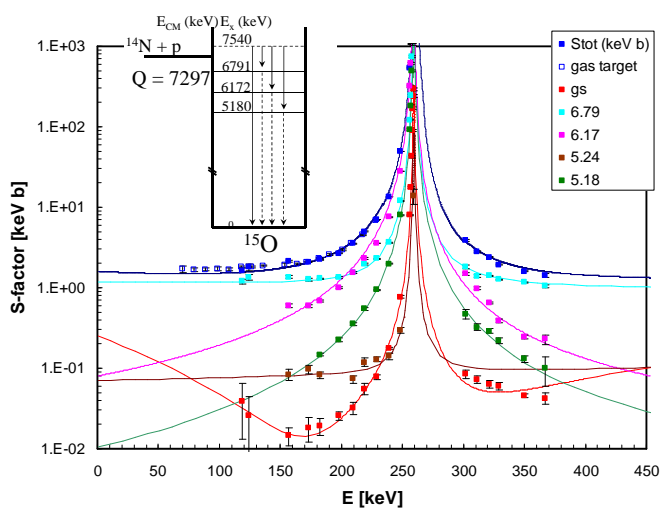

Figure 6: $S$-factor of ${ }^{14} \mathrm{~N}(\mathrm{p}, \gamma){ }^{15} \mathrm{O}$ obtained at LUNA with the high resolution setup [30, 29] (filled symbol) and the high-efficiency setup [33,32] (open symbol). The solid lines is an R-matrix fit from [29].

For temperatures lower than $150 \cdot 10^{6} \mathrm{~K}$ the reaction rate calculated using the LUNA results [6] is a factor of 2 lower with respect to the previous determination [38]. This reduction has several astrophysical implications. In the sun the $\mathrm{CNO}$ cycle contributes to the total energy production only by $2 \%$ instead of $4 \%$ [39], and thus the corresponding neutrino fluxes are reduced accordingly. This is highly relevant for the Borexino neutrino detector [40, 41]. Globular Clusters represent the oldest 
resolved stellar populations. Their age closely coincides with the time elapsed since the epoch of the formation of the first stars in the Universe and provides an independent check on the reliability of cosmological models. With the LUNA determination of the reaction rate, the estimation of the Globular Clusters age is increased by about $1 \mathrm{Gyr}$, i.e. to $14 \pm 1 \mathrm{Gyr}$ [42]. This value is compatible with the model dependent determination by the WMAP experiment [43]. The last consequence is related to the contribution of AGB stars to the the chemical evolution of galaxies. The smaller the ${ }^{14} \mathrm{~N}(\mathrm{p}, \gamma){ }^{15} \mathrm{O}$ reaction rate, the smaller is the helium production rate, and the later the ignition of the He-shell flash. As a consequence the flash will be more violent and the forthcoming dredgeup is more efficient compared with a scenario with a larger ${ }^{14} \mathrm{~N}(\mathrm{p}, \gamma){ }^{15} \mathrm{O}$ reaction rate. Finally, a more efficient dredge-up leads to a larger envelope enrichment, in particular higher ${ }^{12} \mathrm{C}$ and metal abundances, which will pollute the interstellar medium [44].

\subsection{H-shell burning: light nuclei nucleosynthesis}

The proton capture of ${ }^{15} \mathrm{~N}$ is relevant since the compound nucleus ${ }^{16} \mathrm{O}$ can decay through the $\alpha$ particle channel as well as through the $\gamma$-ray channel to the ground state of ${ }^{16} \mathrm{O}$. This introduces a reaction branch linking the first $\mathrm{CNO}$ or $\mathrm{CN}$ cycle with the second $\mathrm{CNO}$, or $\mathrm{NO}$ cycle. The branching has always been a matter of debate since both reactions are characterized by strong low energy resonances [45]. Indirect studies suggest a cross section significantly lower that would translate into a lower rate [46, 47]. High precision experiments performed at higher energies in a wide energy range are extremely important, since they become complementary to low energy data, when direct measurements are not possible. Following this idea a collaboration was started between the Nuclear Science Laboratory at the University of Notre Dame and LUNA. We studied, with the same experimental setup, the ${ }^{15} \mathrm{~N}(\mathrm{p}, \gamma){ }^{16} \mathrm{O}$ reaction over a wide energy range. The energies covered by the experiment range from $2 \mathrm{MeV}$ using the $\mathrm{KN}$ accelerator at $\mathrm{ND}$, down to $100 \mathrm{keV}$ using the $400 \mathrm{kV}$ LUNA accelerator. These studies confirm that the cross section is about a factor of 2 lower than previously thought [38], which translates into a reduced leakage rate from the $\mathrm{CN}$ to the NO cycle in stellar burning [48, 49] (figure 7).

The ${ }^{25} \mathrm{Mg}(\mathrm{p}, \gamma)^{26} \mathrm{Al}$ is of particular importance in connection with satellite observations of a $\gamma$-ray line at $1809 \mathrm{keV}[54,55]$, which arises from the $\beta$-decay of ${ }^{26} \mathrm{Al}$ to ${ }^{26} \mathrm{Mg}\left(\mathrm{T}_{1 / 2}=7 \times 10^{5} \mathrm{yr}\right)$. Moreover, the presence of ${ }^{26} \mathrm{Al}$ in the interstellar medium has been evidenced by the observation of ${ }^{26} \mathrm{Mg}$ isotopic enrichment (extinct ${ }^{26} \mathrm{Al}$ ) in carbonaceous meteorites [56]. Any astrophysical scenario for ${ }^{26} \mathrm{Al}$ nucleosynthesis must be concordant with both observations.

The ${ }^{25} \mathrm{Mg}(\mathrm{p}, \gamma)^{26} \mathrm{Al}$ capture reaction belongs to the $\mathrm{Mg}$ - $\mathrm{Al}$ cycle, that plays a relevant role in the synthesis of $\mathrm{Mg}$ and $\mathrm{Al}$ isotopes and is activated in the H-burning regions of stars, when the temperature exceeds $30-40 \mathrm{MK}$. This condition takes place in H-burning convective cores of the most massive main-sequence stars ( $\mathrm{T}$ up to $50 \mathrm{MK}$ ) [57, 58], in H-burning shells of off-mainsequence stars of any mass ( $T$ up to $10^{8} \mathrm{~K}$ ) $[59,60]$ and in explosive H-burning during Nova-like outbursts ( $T$ up to $4 \cdot 10^{8} \mathrm{~K}$ ) [61]. In particular, the low-energy resonances with $E<200 \mathrm{keV}$ in the ${ }^{25} \mathrm{Mg}(\mathrm{p}, \gamma){ }^{26} \mathrm{Al}$ reaction affect the production of radioactive ${ }^{26} \mathrm{Al}_{g s}$ as well as the resulting $\mathrm{Mg} / \mathrm{Al}$ abundance ratio. The reaction ${ }^{25} \mathrm{Mg}(\mathrm{p}, \gamma){ }^{26} \mathrm{Al}(\mathrm{Q}=6.306 \mathrm{MeV})$ is dominated by narrow resonances. These resonances have been experimentally verified down to a resonance energy of $\mathrm{E}_{R}=190 \mathrm{keV}$ $[62,63]$. From the known level structure of ${ }^{26} \mathrm{Al}$, one expects low-lying resonances at $\mathrm{E}_{R}=93$, 109 , and $130 \mathrm{keV}$, among which the $93 \mathrm{keV}$ resonance appeared most important (figure 8). Also in 


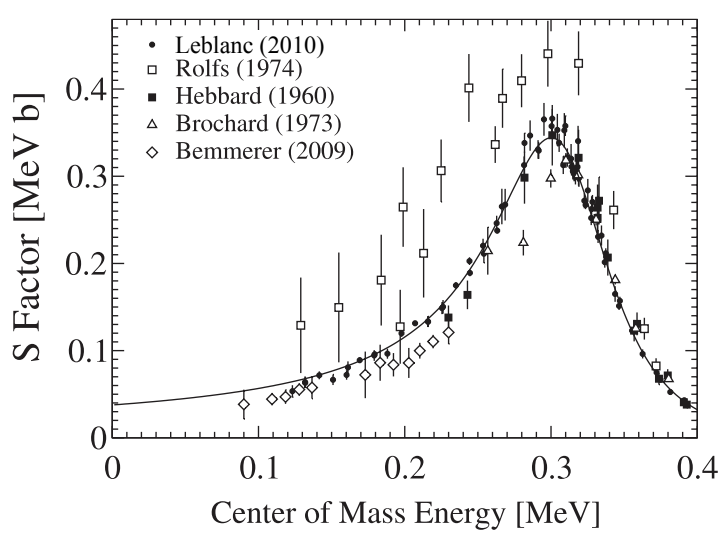

Figure 7: Low-energy $S$-factor of ${ }^{15} \mathrm{~N}(\mathrm{p}, \gamma){ }^{16} \mathrm{O}$. Data of [48] (filled circles) are shown with an AZURE R-matrix calculation along with previous measurements by [50] (open squares), [51] (filled squares), [52] (open triangles), and [53] (open diamonds).

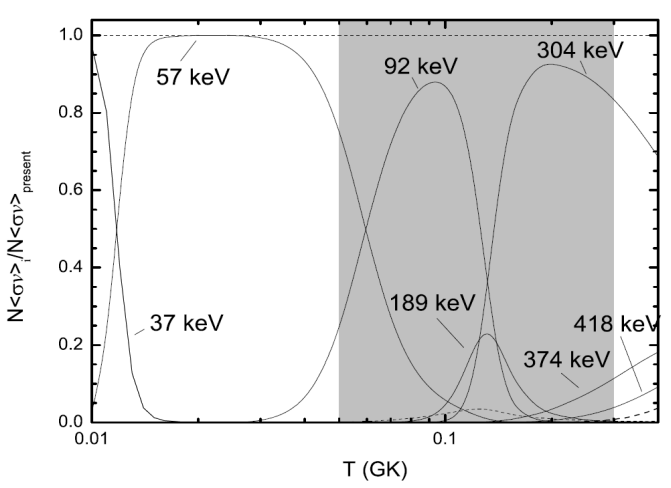

Figure 8: Ratio of individual reaction rate contributions with respect to total recommended rate of ${ }^{25} \mathrm{Mg}(\mathrm{p}, \gamma){ }^{26} \mathrm{Al}$. The contributions of single resonances are indicated, the dashed line refers to the summed contributions of weak resonances as well as resonances above $E=420 \mathrm{keV}$. The grey shaded area represents the range directly investigated at LUNA.

the case of the measurement of ${ }^{25} \mathrm{Mg}(\mathrm{p}, \gamma){ }^{26} \mathrm{Al}$ cross section, the experiment was divided into low efficiency high resolution low and high efficiency resolution phases. The $\gamma$-ray detection setups are similar to those of the ${ }^{14} \mathrm{~N}(\mathrm{p}, \gamma){ }^{15} \mathrm{O}$ experiment.

The study was performed at the LUNA facility using a $4 \pi$ BGO summing crystal. For the first time the $92 \mathrm{keV}$ resonance was directly observed and a resonance strength $\omega \gamma=(2.9 \pm 0.6) \cdot 10^{-10}$ $\mathrm{eV}$ was determined. Additionally, the $\gamma$-ray branchings and strength of the $189 \mathrm{keV}$ resonance were studied with a high resolution HPGe detector yielding an $\omega \gamma$ value in agreement with the BGO measurement, but $20 \%$ larger compared to previous works [64, 65]. On the base of a new experimental study performed at LUNA, it is possible to revise rates of the ${ }^{25} \mathrm{Mg}(\mathrm{p}, \gamma){ }^{26} \mathrm{Al}_{g s}$ and ${ }^{25} \mathrm{Mg}(\mathrm{p}, \gamma){ }^{26} \mathrm{Al}_{m}$. In the temperature range 50 to $150 \mathrm{MK}$, the new recommended rate of the ${ }^{26} \mathrm{Al}_{m}$ production is up to about 4 times higher than previously assumed. In addition, at $T=100 \mathrm{MK}$, the revised total reaction rate is a factor of 2 higher [66].

\subsection{Outlook}

Stimulated by the success of the LUNA experiment several initiatives for installation of a next generation facility have been proposed in the last years. A series of workshops has been organized in Europe (Barcelona 2009, Dresden 2010, Gran Sasso 2011, Canfranc 2012). In its Long Range Plan 2010[67] NUPECC recommends to "fully exploit the currently existing large-scale research infrastructures and (to) perform limited-size upgrades to ensure the best use of the large investments made in the past" and in particular "the nuclear astrophysics underground accelerator LUNA at INFN Gran Sasso, and the exploration of advanced new facilities".

Already 2007 LUNA submitted a Letter of Intent (LOI) to the LNGS scientific committee proposing to measure ${ }^{12} \mathrm{C}(\alpha, \gamma){ }^{16} \mathrm{O}$, and other $(\alpha, \gamma)$ reactions on ${ }^{14,15} \mathrm{~N}$ and ${ }^{18} \mathrm{O}$, which are key reactions of the He burning. In addition the LOI suggested investigations on ${ }^{13} \mathrm{C}(\alpha, n){ }^{16} \mathrm{O},{ }^{22} \mathrm{Ne}(\alpha, n){ }^{25} \mathrm{Mg}$, 
the neutron sources for the s-process. These reactions are relevant at higher temperatures, i.e. larger energies, than reactions belonging to the hydrogen-burning studied so far at LUNA. Therefore the collaboration proposed to install underground a single ended machine with a terminal voltage of approximately 3.5 MV at LNGS. The installation has been approved by the LNGS Scientific Committee in 2010. In summer 2012 the Italian Ministry of Research granted financial support to INFN for site preparation and accelerator installation, first experiments can be expected to start in 2015.

The only other project in a relative advanced stage is in the USA. The Dakota Ion Accelerators for Nuclear Astrophysics project (DIANA) is supported by a collaboration of the University of Notre Dame, University of North Carolina, Western Michigan University, and Lawrence Berkeley National Laboratory. It aims to build a nuclear astrophysics accelerator facility $1.4 \mathrm{~km}$ below ground. The DIANA design [68] foresees to achieve large reaction rates by delivering very high beam currents (up to $100 \mathrm{~mA}$ ) to a high density (up to $10^{18}$ atoms $/ \mathrm{cm}^{2}$ ) super-sonic jet-gas target as well as to solid targets. It will consist of two accelerators, $50-400 \mathrm{kV}$ and $0.4-3 \mathrm{MV}$, that will cover a wide range of ion beam intensities, with sufficient energy overlap to consistently connect the results to measurements above-ground. DIANA will address scientific issues in the field of solar neutrino sources and the metallicity of the sun, carbon-based nucleosynthesis and neutron sources for the production of trans-Fe elements in stars.

\section{References}

[1] R.E. Azuma et al., Phys. Rev. C 81 (2010) 045805

[2] C. Rolfs and W.S. Rodney: Cauldrons in the cosmos, University of Chicago Press (1988)

[3] C. Iliadis, Nuclear Physics of Stars (Weinheim:Wiley-VCH) (2007)

[4] A. Couture et al., Phys. Rev. C 77 (2008) 015802

[5] R.C. Runkle et al., Phys. Rev. Lett. 94 (2005) 082503

[6] H. Costantini, A. Formicola, G. Imbriani, M. Junker, C. Rolfs and F. Strieder, Rep. Prog. Phys 72(2009) 086301

[7] F. Confortola et al., Phys. Rev. C, 75 (2007) 065803

[8] H. V. Klapdor-Kleingrothaus et al., Nucl. Instrum. Methods A 522 (2004) 371

[9] A. Caciolli et al., Eur. Phys. J. A 39 (2009) 179

[10] U. Greife et al., Nucl. Instrum. Methods A 350(1994)327.

[11] R. Bonetti et al., Phys. Rev. Lett. 82(1999)5205

[12] H. Costantini et al., Phys. Lett. B482 (2000)43

[13] A. Formicola et al., Eur. Phys. J. A8 (2000)443

[14] C. Casella et al., Nucl.Phys. A, 706 (2002) 203

[15] A. Formicola, G. Imbriani, M. Junker et al, Nucl. Instrum. Methods A 507(2003)609.

[16] M. Wiescher et al., Annu. Rev. Nucl. Part. Sci. 60(2010)38

[17] B. Aharmin et al, Phys. Rev. C 75(2007)045502

[18] K. Eguchi, Phys. Rev. Lett. 90(2003)021802 
[19] E. Adelberger et al., Rev. Mod. Phys. 83(2011)195

[20] T. A. D. Brown et al., Phys. Rev. C, 76 (2007) 055801

[21] D. Bemmerer et al., Phys. Rev. Lett., 97 (2006) 122502

[22] G. Gyürky et al., Phys. Rev. C, 75 (2007) 35805

[23] H. Costantini et al., Nucl. Phys. A, 814 (2008) 144

[24] A. Di Leva et al., Phys. Rev. Lett., 102 (2009) 232502

[25] P. Descouvemont et al., At. Data Nucl. Data Tables, 88 (2004) 203

[26] T. Kajino et al., ApJ, 319 (1987) 531

[27] K. M. Nollett, Phys. Rev. C, 63 (2001) 054002

[28] M. Wiescher, J. Görres, E. Ubersederet, G. Imbriani and M. Pignatari, Annu. Rev. Nucl. Part. Sci. 60 (2010) 381

[29] G. Imbriani et al., Eur. Phys. J. A, 25 (2005) 455-466

[30] A. Formicola et al., Phys. Lett. B, 591 (2004) 61

[31] M. Marta et al., Phys. Rev. C, 78 (2008) 022802(R)

[32] D. Bemmerer et al., Nucl. Phys. A, 779 (2006) 297

[33] A. Lemut et al., Phys. Lett. B, 634 (2006) 483L

[34] P.F. Bertone et al., Phys. Rev. Lett., 87 (2001) 152501

[35] D.Schürmann et al., Phys. Rev. C, 77 (2008) 055803

[36] K. Yamada et al., Phys. Lett. B, 579 (2004) 265

[37] A.M. Mukhamedzhanov et al., Phys. Rev. C, 67 (2003) 065804

[38] C. Angulo et al., Nucl. Phys. A 656 (1999) 3

[39] S. Degl'Innocenti et al., Phys. Lett. B, 590 (2004) 13

[40] C. Arpesella et al., Phys. Lett. B, 658 (2008) 101

[41] C. Arpesella et al., Phys. Rev. Lett., 101 (2008) 091302

[42] G. Imbriani et al., A\&A, 420 (2004) 625

[43] D. N. Spergel et al., ApJ Suppl. Ser., 170 (2007) 377

[44] F. Herwig, S. Austin and J. C. Lattanzio, Phys. Rev. C, 73 (2006) 025802

[45] A. Redder et al., Z. Phys. A, 305 (1982) 325

[46] A.M. Mukhamedzhanov et al., Phys. Rev. C, 78 (2008) 015804

[47] M. La Cognata et al., Phys. Rev. C, 80 (2009) 012801

[48] P.J. Leblanc et al., Phys. Rev. C, 82 (2010) 055804

[49] G. Imbriani et al., Phys. Rev. C, 85 (2012) 065810

[50] C. Rolfs and W. S. Rodney, Nucl. Phys. A 235, 450 (1974).

[51] D. Hebbard, Nucl. Phys. 15, 289 (1960). 
[52] F. Brochard, P. Chevallier, D. Disdier, and F. Scheibling, J. Phys. 34, 363 (1973).

[53] D. Bemmerer et al., J. Phys. G 36, 045202 (2009).

[54] J. Knödlseder, D. Dixon, K. Bennett et al., A\&A, 345 (1999) 813

[55] C. Winkler, T.C. Courvoisier, G. Di Cocco et al., A\&A, 411 (2003) L1

[56] G.J. Wasserburg, in Protostars and planet II, edited by D.C. Black and M.S. Matthews (Universirty of Arizona Press, Tucson, 1985), p.703

[57] A. Palacios, G. Meynet et al., Astron. Astrophys. 429 (2005) 613.

[58] M. Limongi, A. Chieffi, Astrophys. J. 647 (2006) 483.

[59] R.M. Cavallo, A.V. Sweigart, R.A. Bell, Astrophys. J. 492 (1998) 575.

[60] [4] R.G. Izzard et al., Astron. Astrophys. 466 (2007) 641.

[61] [5] C. Iliadis, A. Champagne et al., Astrophys. J. Suppl. 142 (2002) 105.

[62] A.E. Champagne et al., Nucl. Phys. A 505 (1989) 384.

[63] D.C. Powell, C. Iliadis et al., Nucl. Phys. A 644 (1998) 263.

[64] B. Limata et al., Phys. Rev. C, 82 (2010) 01580.

[65] F. Strieder et al., Phys. Lett. B 707 (2012) 60.

[66] O. Straniero et al., submitted to ApJ.

[67] European Science Foundation, "Perspectives of Nuclear Physics in Europe NuPECC Long Range Plan 2010", www.nupecc.org/pub/hb12/hb2012.pdf

[68] A. Lemut et al. Phys. Rev. ST Accel. Beams 14 (2011) 100101 